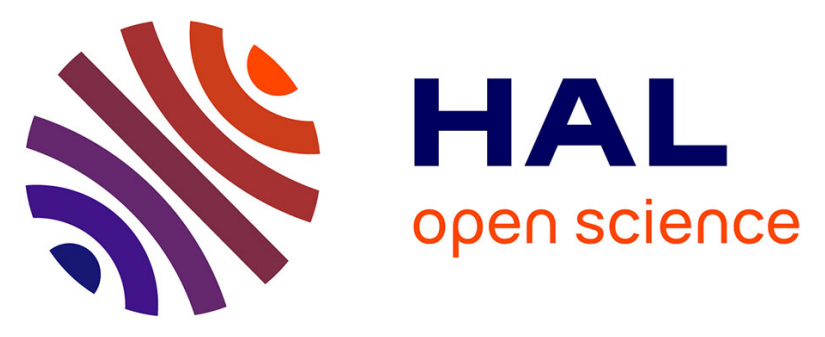

\title{
Hypoxia enhances innate immune activation to Aspergillus fumigatus through cell wall modulation
}

Kelly M Shepardson, Lisa y Ngo, Vishukumar Aimanianda, Jean-Paul Latge, Bridget M Barker, Sara J Blosser, Yoichiro Iwakura, Tobias Hohl, Robert A Cramer

\section{To cite this version:}

Kelly M Shepardson, Lisa y Ngo, Vishukumar Aimanianda, Jean-Paul Latge, Bridget M Barker, et al.. Hypoxia enhances innate immune activation to Aspergillus fumigatus through cell wall modulation. Microbes and Infection, 2013, 15 (4), pp.259-269. 10.1016/j.micinf.2012.11.010 . pasteur-02521060

\section{HAL Id: pasteur-02521060 \\ https://hal-pasteur.archives-ouvertes.fr/pasteur-02521060}

Submitted on 27 Mar 2020

HAL is a multi-disciplinary open access archive for the deposit and dissemination of scientific research documents, whether they are published or not. The documents may come from teaching and research institutions in France or abroad, or from public or private research centers.
L'archive ouverte pluridisciplinaire HAL, est destinée au dépôt et à la diffusion de documents scientifiques de niveau recherche, publiés ou non, émanant des établissements d'enseignement et de recherche français ou étrangers, des laboratoires publics ou privés.

$$
\text { Copyright }
$$




\title{
Original article
}

\section{Hypoxia enhances innate immune activation to Aspergillus fumigatus through cell wall modulation}

\author{
Kelly M. Shepardson ${ }^{\mathrm{a}, 2}$, Lisa Y. Ngo ${ }^{\mathrm{b}}$, Vishukumar Aimanianda ${ }^{\mathrm{c}}$, Jean-Paul Latgé ${ }^{\mathrm{c}}$, \\ Bridget M. Barker ${ }^{a}$, Sara J. Blosser ${ }^{\text {a }}$, Yoichiro Iwakura ${ }^{\mathrm{d}, 3}$, Tobias M. Hohl ${ }^{\mathrm{b}, * *, 1}$, \\ Robert A. Cramer ${ }^{\mathrm{a}, *, 1}$ \\ ${ }^{a}$ Department of Immunology and Infectious Diseases, Montana State University, Bozeman, MT 59717, USA \\ ${ }^{\mathrm{b}}$ Vaccine and Infectious Disease Division, Clinical Research Division, Fred Hutchinson Cancer Research Center, Seattle, WA 98109, USA \\ ${ }^{\mathrm{c}}$ Unité des Aspergillus, Institut Pasteur, Paris 75015, France \\ ${ }^{\mathrm{d}}$ Research Institute for Biomedical Sciences, Tokyo University of Science, Noda, Chiba 278-0022, Japan
}

Received 31 August 2012; accepted 23 November 2012

Available online 4 December 2012

\begin{abstract}
Infection by the human fungal pathogen Aspergillus fumigatus induces hypoxic microenvironments within the lung that can alter the course of fungal pathogenesis. How hypoxic microenvironments shape the composition and immune activating potential of the fungal cell wall remains undefined. Herein we demonstrate that hypoxic conditions increase the hyphal cell wall thickness and alter its composition particularly by augmenting total and surface-exposed $\beta$-glucan content. In addition, hypoxia-induced cell wall alterations increase macrophage and neutrophil responsiveness and antifungal activity as judged by inflammatory cytokine production and ability to induce hyphal damage. We observe that these effects are largely dependent on the mammalian $\beta$-glucan receptor dectin- 1 . In a corticosteroid model of invasive pulmonary aspergillosis, A. fumigatus $\beta$-glucan exposure correlates with the presence of hypoxia in situ. Our data suggest that hypoxia-induced fungal cell wall changes influence the activation of innate effector cells at sites of hyphal tissue invasion, which has potential implications for therapeutic outcomes of invasive pulmonary aspergillosis.
\end{abstract}

(C) 2012 Institut Pasteur. Published by Elsevier Masson SAS. All rights reserved.

\footnotetext{
* Corresponding author. Current address: Department of Microbiology and Immunology, Geisel School of Medicine at Dartmouth, Hanover, NH 03755, USA. Tel.: +16036501040.

** Corresponding author. Clinical Research Division, Fred Hutchinson Cancer Research Center, E.D. Thomas Building, Room D3-367, Seattle, WA 98109, USA.

E-mail addresses: thohl@fhcrc.org (T.M. Hohl), Robert.A.Cramer.Jr@ dartmouth.edu (R.A. Cramer).

1 These authors contributed equally to this manuscript.

2 Current address: Department of Microbiology and Immunology, Geisel School of Medicine at Dartmouth, Hanover, NH 03755, USA.

${ }^{3}$ CREST.
}

\section{Introduction}

Over the past three decades increased use of medical technologies such as organ transplantation, chemotherapy, and other immunomodulation therapies, have led to an increase in the incidence of human fungal infections [1]. In patients undergoing these therapies, the mold Aspergillus fumigatus remains one of the most common causes of fungal morbidity and mortality [2]. Despite the advent and increased prophylactic use of antifungal drugs, the mortality and morbidity of Aspergillus infections, particularly with invasive pulmonary aspergillosis (IPA), remains high [2-4]. One avenue of research that holds promise for improving invasive fungal infection (IFI) patient outcomes is the study of fungal-host 
interactions. A better understanding of the pathogenesis and physiology of both the fungus and host as they interact in vivo may identify new therapeutic strategies that can augment existing antifungal drug regimens.

A critical step in understanding fungal-host interactions is to elucidate the micro-environmental stresses encountered by both the pathogen and host cells as they interact in situ in dynamic microenvironments. Although pathogenic fungi respond to temperature, reactive oxygen intermediates, $\mathrm{pH}$ changes, and macro- and micronutrient limitation [5-9], much less is known about how human pathogenic fungi respond to oxygen limitation, particularly when hypoxic conditions are encountered in host tissue. Moreover, how infection-induced changes in tissue microenvironments alter host immune responses are poorly defined. We recently observed that host and fungal cells encounter hypoxic microenvironments in the lung in murine models of IPA [10]. Oxygen is required for survival of both the fungus and host cells and when oxygen levels are insufficient for normal cellular processes a significant stress is placed on obligate aerobic eukaryotic organisms. Oxygen is important for ATP production through oxidative phosphorylation, sterol production, fatty acid synthesis, the TCA cycle, and other pathways that are involved in cell metabolism and growth. Adaptation to hypoxia is a requirement for A. fumigatus in vivo survival based on the need for the sterol regulatory element binding protein, SrbA, to overcome hypoxia and cause disease in murine models of IPA $[11,12]$.

To control and overcome infection, the host must be able to recognize the pathogen and mount a robust immune response. For fungi, a major source of innate immune system recognition is the fungal cell wall. The cell wall of A. fumigatus is composed of different polysaccharides that are comprised into a fibrillar skeleton made up of $\beta$-glucan and chitin and an amorphous cement containing galactomannan and $\alpha$-glucan $[13,14]$. These fractions are located on the outside of the plasma membrane and are involved in host recognition as fungal pathogen associated molecular patterns (PAMPs) [15]. In addition, galactosaminogalactan is secreted by A. fumigatus and covers the cell wall surface and is also present in the ECM with immunomodulatory activity [16]. Flux of critical pathways involved in the synthesis of these cell wall and cell membrane components, such as glycolysis and fatty acid synthesis pathways was demonstrated to occur in response to hypoxia $[7,12,17-23]$. Detection of fungal cell wall components drives innate and adaptive immune activation that can either lead to protective responses or host mediated tissue damage [24]. Exposure of these PAMPs in the fungal cell wall, either through environmental, nutritional or drug stress, has been demonstrated to be important to initiate immune responses [7,14,24,25]. Previously, exposure of A. fumigatus to echinocandin drugs, $\beta$ - $(1,3)$-glucan synthase inhibitors, was shown to increase exposure of $\beta$ - $(1,3)$-glucan on the surface of hyphae leading to an increased dectin-1-dependent inflammatory response and antifungal activity, with this effect being Aspergillus-specific [25,26]. Dectin-1 is a major fungal pattern recognition receptor (PRR) that recognizes soluble $\beta$ - glucan [27,28]. For fungi, stress leads to increased $\beta$-glucan levels and here we report that hypoxia is not an exception. Previous studies have suggested that levels of oxygen can influence the nature and abundance of potential immunomodulatory factors in fungi including the cell wall proteome of Candida albicans and cell wall composition in Saccharomyces cerevisiae $[17,22]$. In our most recent studies, we have demonstrated that hypoxia also causes alterations of the cell wall transcriptome of A. fumigatus, specifically, the transcriptional up-regulation of a subset of genes involved in $\beta$ glucan and chitin synthesis [18]. The outcome of these hypoxia-mediated alterations may be of clinical significance as most antifungal drug targets are components of the cell wall and plasma membrane, whose levels are altered by oxygen availability.

How hypoxia-induced alterations in the cell wall affect the host immune system responses to and recognition of $A$. fumigatus is poorly understood. A further understanding of the mechanisms involved may provide new insights into management and treatment of IFIs. For example, increased PAMP exposure may contribute to immune reconstitution syndrome (IRIS) [29]. IRIS involves an exaggerated response of host effector cells to pathogens or failure in activation of regulatory effectors to dampen the response [30]. Un-masking of $\beta$-glucan following antifungal treatment has been speculated to illicit IRIS due to increased inflammatory cytokine release. Clinical onset of IRIS in neutropenic patients with IPA may occur following neutrophil recovery, resulting in worsening of clinical symptoms, morbidity, and mortality [31]. Therefore, the significance of PAMPs alterations that occur in vivo and how this affects immune effector responses is of critical importance.

In this study, we observe that lung hypoxic conditions found in the corticosteroid model of IPA has a profound impact on the A. fumigatus cell wall composition and the resulting activation of phagocytic effector cells. Hypoxia in vitro and in vivo leads to increased exposure of fungal $\beta$ glucan, the major PAMP for dectin-1, and an overall increase in cell wall mass and diameter. Levels of other immunomodulatory cell wall components are also altered in response to hypoxia. In turn, hypoxia-grown hyphae trigger enhanced dectin-1-dependent activation of macrophages and neutrophils, suggesting a mechanism by which tissue hypoxia may act to direct and concentrate phagocyte responses at sites of tissue invasion by exploiting obligate fungal adaptation to in vivo infection site microenvironments.

\section{Materials and methods}

\subsection{Fungal cultures and growth conditions}

A. fumigatus strain CBS144.89 was used in all experiments, but strain AF293 was used in the ELISA experiments to determine whether the observed effects were fungal strain specific. All strains were grown on glucose minimal medium with $1.5 \%$ agar at $37{ }^{\circ} \mathrm{C}$. Conidia were dislodged from plates with a cell scrapper, re-suspended in $0.01 \%$ Tween-20, and 
filtered through miracloth (EMD chemicals, CalBiochem). All strains were routinely grown in tissue culture (TC) medium (RPMI, 10\% FBS, $5 \mathrm{mM}$ HEPES buffer, $1.1 \mathrm{mM}$ L-glutamine, $0.5 \mathrm{U} / \mathrm{ml}$ penicillin, and $50 \mathrm{mg} / \mathrm{ml}$ streptomycin) at $37^{\circ} \mathrm{C}$, unless otherwise specified.

\subsection{Hypoxic cultivation}

Strains were grown in TC medium at $37{ }^{\circ} \mathrm{C}$. Normoxic conditions were considered general atmospheric levels within the lab $(\sim 21 \%)$. Hypoxic conditions were as follows: the $\mathrm{O}_{2}$ set point was $1 \%$ and the $\mathrm{CO}_{2}$ set point was $5 \%$. Oxygen levels were maintained with $94 \% \mathrm{~N}_{2}$ and a gas regulator. Two different devices were used to maintain hypoxic environments, a Biospherix C-Chamber with $\mathrm{O}_{2}$ levels controlled by a PROOx controller and $\mathrm{CO}_{2}$ levels controlled with $\mathrm{PRO}-\mathrm{CO}_{2}$ controller (Biospherix, Lacona, NY, USA) and an $\mathrm{INVIVO}_{2}$ 400 Hypoxia Workstation (Ruskinn Technology Limited, Bridgend, UK). Additionally, experiments, where stated, were also conducted using the AnaeroPack System with anaerobic packets. No significant differences were observed in pilot experiments between the different hypoxia cultivation methods.

\subsection{Soluble dectin-1}

A. fumigatus conidia were adhered for $7 \mathrm{~h}$ and $8 \mathrm{~h}$ under normoxic and hypoxic $\left(1 \% \mathrm{O}_{2}, 5 \% \mathrm{CO}_{2}, 94 \% \mathrm{~N}_{2}\right.$ or using sealed AneroPack system with anaerobic packet (data not shown)) conditions, respectively, to sterile, 8-chamber well glass slides. The hyphae were UV irradiated, blocked, and stained with conditioned medium containing s-dectin-hFc followed by DyLight 594-conjugated, goat anti-human IgG1 [32]. After being washed, the glass slides were mounted by coverslips with Prolong mounting medium (Molecular Probes) and were captured using an Olympus Deltavision microscope. Dectin-1 staining was measured by averaging the amount of edge staining, detected by the program, above the signal threshold to the total area of each hypha. All hyphal measurements were conducted using ImageJ software (version 1.45).

\subsection{Transmission electron microscopy}

Fungal cells were grown in 8-well chamber slides (LabTek, Naperville, Illinois, United States) in normoxic or hypoxic (in sealed AnaeroPack System with anaerobic packet or hypoxic cultivation (data not shown), described above) conditions and UV-inactivated. For TEM, hyphae were fixed in $1 / 2$ Karnovsky's fixative (2\% paraformaldehyde, $2.5 \%$ glutaraldehyde buffered with $0.2 \mathrm{M}$ cacodylate buffer) and postfixed in $1 \%$ osmium tetroxide in $0.1 \mathrm{M}$ cacodylate buffer, dehydrated with ethanol and embedded in Epon 812 for $48 \mathrm{~h}$ at $60{ }^{\circ} \mathrm{C}$. Sectioned hyphae were further stained with uranyl acetate for $2 \mathrm{~h}$ and lead citrate for $5 \mathrm{~min}$. Samples were imaged using JEOL1230 transmission electron microscope. Hyphal diameters and cell wall widths were measured using ImageJ software (version 1.45).

\subsection{Nucleic acid extraction}

Tissue was re-suspended in Trizol reagent and chloroform to extract RNA. Tubes were centrifuged at $16,000 \times g$ for $15 \mathrm{~min}$ at $4{ }^{\circ} \mathrm{C}$. The clear upper layer was further extracted with an equal volume of $80 \% \mathrm{EtOH}$. Samples were applied to RNeasy spin column (Qiagen RNA kit) following manufacturer's instructions. RNA was eluted with RNase free water.

\subsection{Real-time RT-PCR}

RNA was DNase treated with DNA-free kit (Ambion) and reverse transcribed with QuantiTect reverse transcription kit (Qiagen, USA). Primers for all genes of interest were designed with PrimerQuest (IDT) and manufactured by IDT, USA. Sequences are: $f k s A$, Fwd-TGTTCCTTGTCCTTCTCATT GCCC, Rev-TGTTGTCCTTGTCAAGAGGCTGGA, and tefA, Fwd-GTGACTCCAAGAACGATCCC, Rev-AGAACT TGCAAGCAATGTGG. All reactions were performed on BioRad MyIQ real-time PCR detection system with IQ SYBR green supermix (BioRad, Hercules, CA). The $\Delta \Delta C_{t}$ method was used to assess changes in mRNA abundance, using tefA as the housekeeping gene [33]. Results presented are the mean and standard deviation from 3 biological replicates.

\subsection{Cell wall analysis}

A. fumigatus was grown in liquid GMM under normoxic conditions for $6.5 \mathrm{~h}$ until swelling and then transferred to new flasks to grow under either normoxic or hypoxic conditions for $24 \mathrm{~h}$. Mycelia were collected by filtration, washed with sterile water, flash frozen, lyophilized, and disrupted in a FastPrep-24 cell homogenizer (MP Biomedicals, United States) at $4{ }^{\circ} \mathrm{C}$ using $0.5 \mathrm{~mm}$ glass beads and the disrupted mycelia were centrifuged (4000 rpm, $10 \mathrm{~min}$ ) to isolate the cell wall. The monosaccharide composition (percent) of the cell wall polysaccharides was determined as previously described [34]. Results presented are the mean and standard deviation from 5 biological replicates.

\subsection{ELISA}

Bone marrow (BM) cells were eluted from tibias and femurs of $8-12$ week old C57BL/6 or dectin- $1^{(-1-)}$ mice, lysed of red blood cells, and cultured for macrophages in RP20 (RPMI, 20\% FCS, 5 mM HEPES buffer, $1.1 \mathrm{mM}$ L-glutamine, $0.5 \mathrm{U} / \mathrm{ml}$ penicillin, and $50 \mathrm{mg} / \mathrm{ml}$ streptomycin) supplemented with $30 \%$ (v/v) L929 cell supernatant (source of MCSF) or neutrophils in murine neutrophil buffer (HBSS containing $0.1 \%$ FBS and $1 \%$ glucose). BM cells for macrophages were plated in a volume of $20 \mathrm{ml}$ at a density of $2.5 \times 10^{6}$ cells $/ \mathrm{ml}$ in $10 \mathrm{ml}$ petri dishes. The medium was exchanged on day 3. Adherent BM-derived macrophages (BMDMs) were harvested on day 6. BM cells for neutrophils (BMDNs) were suspended in $3 \mathrm{ml} 45 \%$ percoll and isolated from a 30 min $1600 \times g$ percoll gradient (top to bottom: $3 \mathrm{ml}$

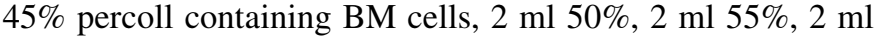


$62 \%$, and $3 \mathrm{ml} 81 \%$ ) in an Allegra $\mathrm{X}-15 \mathrm{r}$ benchtop centrifuge, SX4750A rotor (Bechman Coulter). BMDNs were collected from the $62 / 81 \%$ border and washed with HBSS. In both cases, cells were washed and plated in $0.2 \mathrm{ml} \mathrm{TC}$ medium at a density of $5 \times 10^{5}$ cells $/ \mathrm{ml}$ in 96 well plates and stimulated for $18 \mathrm{~h}$ with UV-irradiated hyphae $\left(2 \times 10^{4} /\right.$ well $)$ grown for $18 \mathrm{~h}$ in normoxic or hypoxic (in sealed AnaeroPack System with anaerobic packet or hypoxic cultivation, described above) conditions. After $18 \mathrm{~h}$ of co-culture, supernatants were collected for ELISA. Commercially available ELISA kits for TNF (eBioscience, San Diego, California, United States) and CXCL2 (R\&D Systems, Minneapolis, Minnesota, United States) were used according to the manufactures' instructions. The limit of detection was $15 \mathrm{pg} / \mathrm{ml}$ for both TNF and CXCL2.

\subsection{Ethics statement}

This study was carried out in strict accordance with the recommendations in the Guide for the Care and Use of Laboratory Animals of the National Institutes of Health. The animal experimental protocol was approved by the Institutional Animal Care and Use Committee (IACUC) at Montana State University (Federal-Wide Assurance Number: A363701). Heparinized venous blood samples from healthy donors were collected in accordance with a protocol approved by the Institutional Review Board for Human Subjects, National Institute of Allergy and Infectious Diseases, and Montana State University. All donors provided written consent to participate in the study.

\subsection{XTT assay}

J774.1 and RAW264.7 macrophage (MФ)-like cell lines, primary human neutrophils (PMNs), and peritoneal macrophages were incubated with $A$. fumigatus germlings, grown at $30{ }^{\circ} \mathrm{C}$ for $16 \mathrm{~h}$ and transferred to $37^{\circ} \mathrm{C}$ for 2 additional hrs, in a 9:1 (effector:target) ratio under normoxic and hypoxic conditions for $2 \mathrm{~h}$ (J774.1, RAW264.7, and PMNs) or $5 \mathrm{~h}$ (peritoneal macrophages) [35,36]. Following incubation, cells were cold water lysed and the remaining alive germlings were quantitated by measuring the OD at $450 \mathrm{~nm}$ following a $1 \mathrm{~h}$ incubation with $400 \mu \mathrm{L}$ of $0.4 \mathrm{mg} / \mathrm{ml}$ XTT (2,3-bis(2methoxy-4-nitro-5-[(sulphenylamino)carbonyl)]-2 $\mathrm{H}$-tetrazolium-hydroxide) solution with $0.5 \mathrm{mg} / \mathrm{ml}$ of the electroncoupling agent coenzyme $\mathrm{Q}$. The percent fungal damage was defined by the equation: $\left(1-\left[A_{450}\right.\right.$ of fungi with $\mathrm{M} \Phi-A_{450}$ of $\mathrm{M} \Phi$ alone $] /\left[A_{450}\right.$ of fungi alone $\left.]\right) \times 100$. This equation eliminates any artifact from the $\mathrm{M \Phi s}$ and therefore is a measure of metabolically active fungi. The metabolic activity of normoxic grown hyphae increased at $5 \mathrm{~h}$ compared to hypoxic grown hyphae. This is accounted for in the assay, as hyphal damage was determined for each condition separately. Human PMNs were isolated from whole blood in endotoxinfree conditions $(<25.0 \mathrm{pg} / \mathrm{ml})$, as described previously in [37]. Cell viability and purity of preparations were assessed by flow cytometry (LSRII; BD Biosciences). Cell preparations contained $\sim 99 \%$ PMNs. Peritoneal macrophages were isolated from naive C57BL/6 and dectin-1 KO mice (8-12 weeks) with a peritoneal wash of $5 \mathrm{ml}$ of PBS.

\subsection{In vivo hypoxia and dectin-1 immunostaining}

Staining was carried out on the triamcinolone (corticosteroid) murine model of IPA as previously described in Grahl et al. [10]. Briefly, unanesthetized mice inhaled $40 \mathrm{ml}$ of an aerosolized suspension of $1 \times 10^{9}$ conidia/ml of A. fumigatus, while control mice inhaled $40 \mathrm{ml}$ of aerosolized $0.01 \%$ Tween 80 in a Hinners inhalational chamber for $45 \mathrm{~min}$ as previously described [38]. At set time point's mice were intravenously injected with hypoxyprobe at a dose of $60 \mathrm{mg} / \mathrm{kg}$ (Hypoxyprobe Inc., Burlington, MA, USA). After 60-90 min, mice were sacrificed by pentobarbital anesthesia $(100 \mu \mathrm{g} / \mathrm{g}$ body weight) followed by exsanguination. The lungs of each mouse were filled with OCT (frozen tissue matrix), separated, and frozen in liquid nitrogen after embedding with OCT. Lungs were cryosectioned into $5 \mu \mathrm{m}$ sections. Slides were fixed in cold acetone for $15 \mathrm{~min}$, washed (PBS, $2 \times 5 \mathrm{~min}$ ) and blocked with normal serum block (NSB: PBS $+10 \%$ goat serum $+1.25 \%$ mouse serum) at room temperature for $30 \mathrm{~min}$. Sections were washed and incubated overnight with the mouse monoclonal antibody FITC-Mab1 (Hypoxyprobe-1 Plus Kit, Hypoxyprobe Inc., Burlington, MA, USA) diluted 1:400 in NSB and with a 1:4 dilution of conditioned medium containing s-dectin-hFc. Aspergillus isotype control slides were incubated only with FITC-Mab1 and hypoxyprobe isotype control slides only with s-dectin-hFc (data not shown). Following another wash, sections were incubated with DyLight 594-conjugated mouse anti-FITC (Jackson ImmunoResearch Laboratories, West Grove, PA) and AlexaFluor488-conjugated goat anti-human (Invitrogen, Carlsbad, CA, USA) diluted 1:400 in NSB for 60 min room temperature. Sections were washed a final time before being mounted with prolong Gold antifade reagent with DAPI (Invitrogen, Carlsbad, CA, USA). Analysis of the sections was performed using the Nikon Eclipse 80i microscope and SPOT imaging system (Nikon Instruments Inc., Melville, NY, USA). Quantification of staining was determined using intensity plots in the Spot software. Two to four mice were examined. Mean fluorescence intensity (MFI) of staining was determined using two separate images. Hypoxyprobe and dectin-1 staining was quantified in locations of hypoxia and normoxia (based on hypoxyprobe staining level). The signal threshold was adjusted to same level for both colors and the MFI was detected above the signal threshold. For each image, 5 locations of hypoxia and normoxia were each analyzed. All MFI measurements were conducted using ImageJ software (version 1.45).

\section{Results}

\subsection{Hypoxia induces alterations in the cell wall and hyphal morphology of Aspergillus fumigatus}

We previously reported the existence of hypoxic microenvironments and the need for A. fumigatus to adapt to these 
conditions for in vivo growth in murine models of IPA [10,11]. Recently it has been demonstrated that in response to multiple extracellular stresses, such as oxygen limitation and antifungal drugs, the composition and structure of the fungal cell wall is altered $[17,21,22]$. We therefore sought to determine how hypoxia affected the morphology and cell wall of A. fumigatus hyphae. Initial light microscopy-based observations revealed that A. fumigatus hypoxic growth leads to morphological changes in hyphal extension and hyphal width (data not shown). Importantly, these changes were also observed in vivo at sites of A. fumigatus infection in the lung 3 and 4 days post-infection, when tissue hypoxia is a prominent finding in corticosteroidtreated mice [10]. To further examine the hypoxia-induced changes in hyphal morphology, normoxia- and hypoxiagrown hyphae were analyzed by transmission electron microscopy (TEM). TEM images revealed that hypoxia leads to an increase in the cell wall width and a decrease in overall hyphal diameter (Fig. 1). An electron dense layer on the periphery of the cell wall was also substantially increased in hypoxic grown hyphae (Fig. 1B). Thus, hypoxia leads to significant changes in the fungal cell wall.

\subsection{Hypoxia induces increased $\beta$-glucan synthase transcription and $\beta$-glucan surface exposure}

We next asked the question how the overall cell wall composition is affected by exposure to hypoxia. Previously, we observed increased transcript levels of genes encoding proteins involved in cell wall and cell membrane synthesis in a wholegenome transcript profile of the A. fumigatus hypoxia response [18]. Therefore, we first sought to determine if the stress of hypoxia would result in changes in $\beta$-glucan composition in the cell wall. The results in Fig. 2(A and B) show that exposure of A. fumigatus to hypoxic conditions causes increased binding of soluble dectin-1 to the hyphae of A. fumigatus compared to normoxic grown hyphae [39]. In addition to increases in soluble dectin-1 binding to hypoxic hyphae, transcript levels of $f k s A$, the 1,3- $\beta$-glucan synthase catalytic subunit, were increased in hyphae exposed to increasing amounts of time in hypoxia consistent with our previously reported wholegenome transcript profiling data (Fig. 2C). Thus, hypoxia causes an increase in $\beta$-glucan synthase transcript levels and increased exposure of $\beta$-glucan on the hyphal surface.
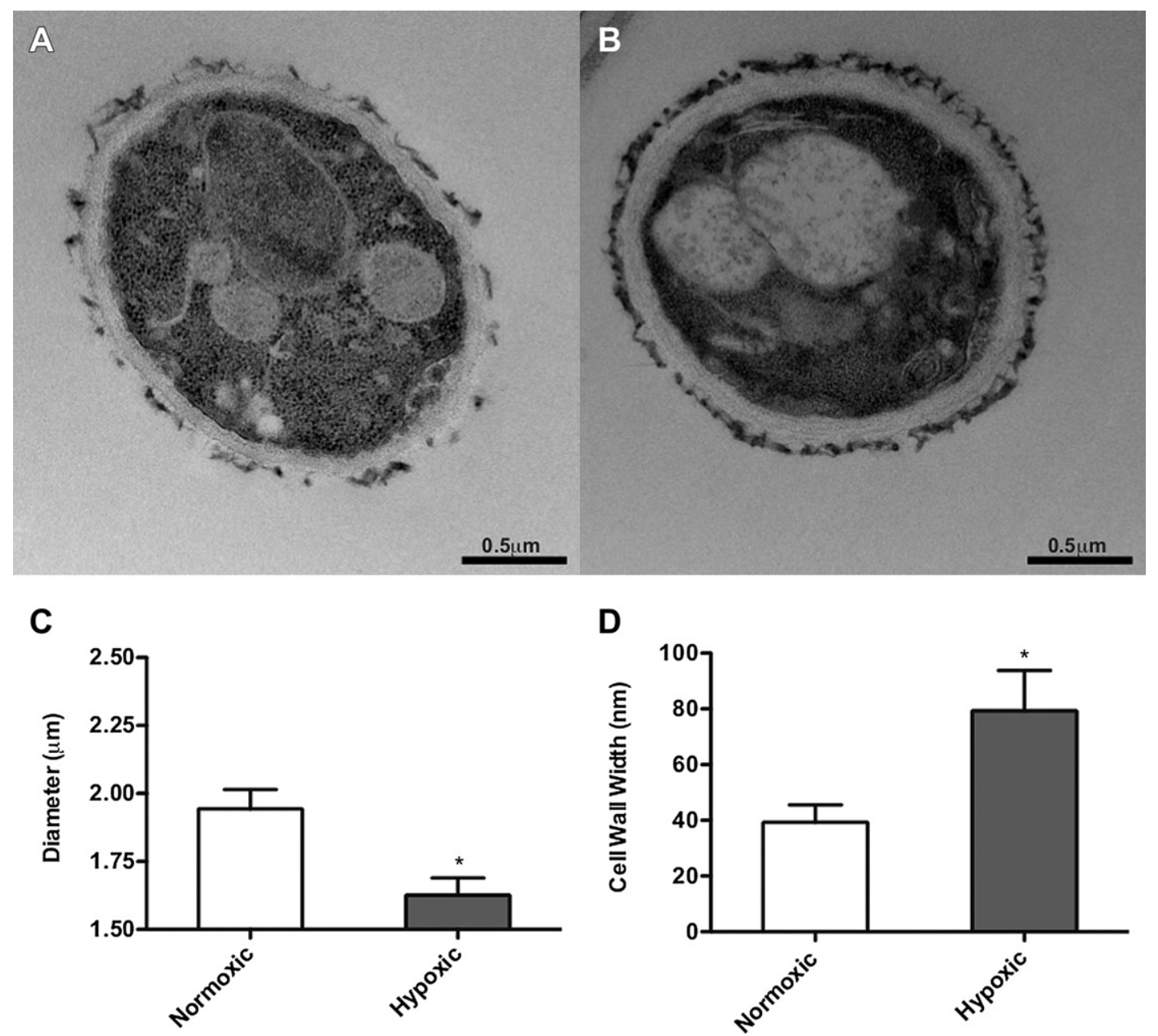

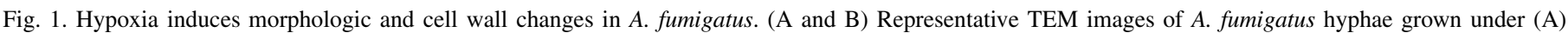

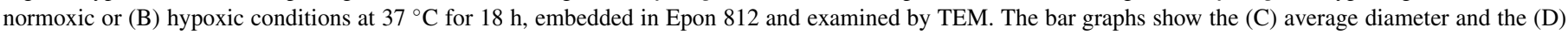

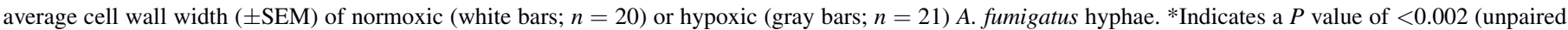
two-tailed Students $t$ test). 

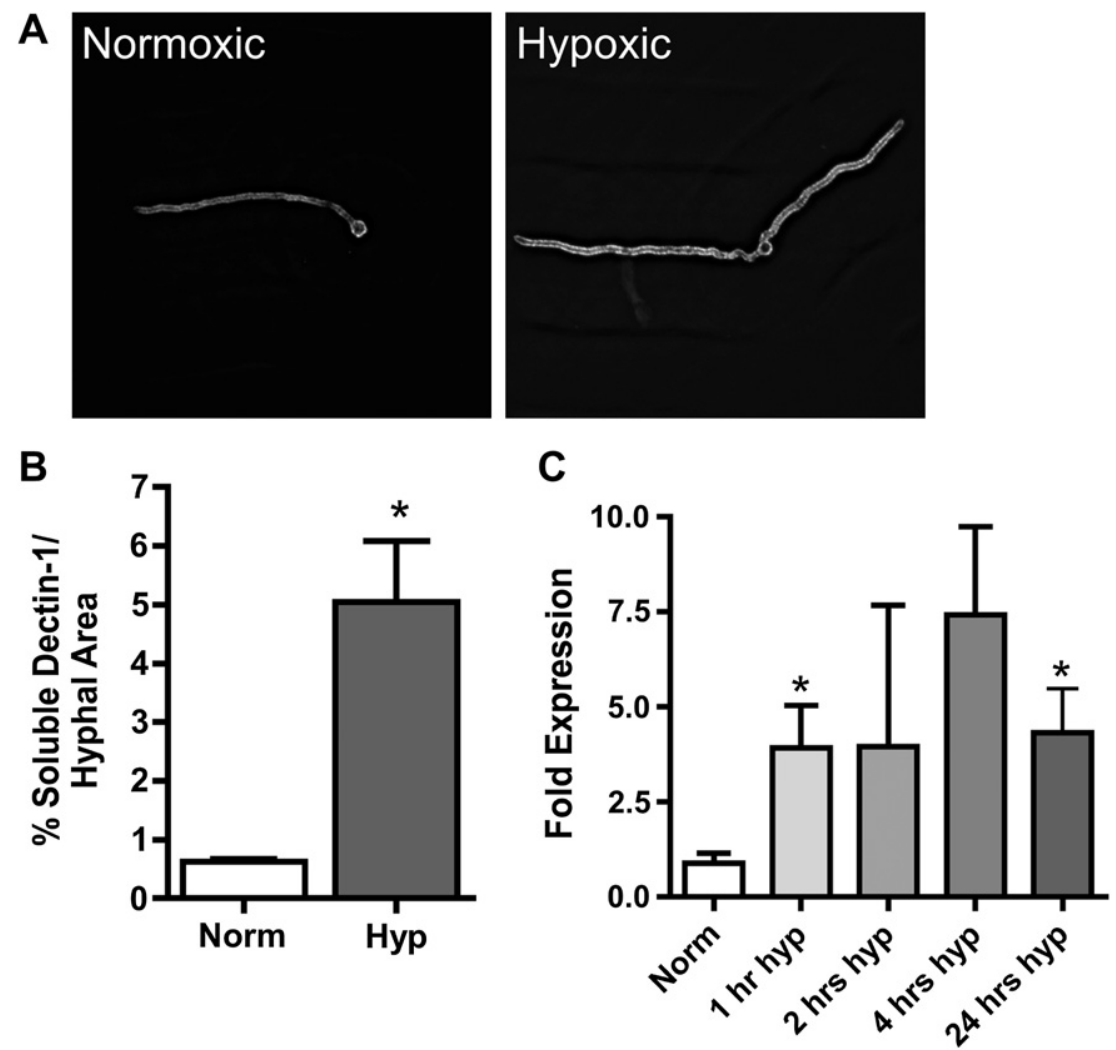

Fig. 2. Hypoxia induces an increase in $\beta$-glucan exposure and $f k s A$ transcript levels. (A) A. fumigatus was grown under normoxic and hypoxic $\left(1 \% \mathrm{O}_{2}, 5 \% \mathrm{CO}_{2}\right)$ conditions for $16 \mathrm{~h}$ until they formed hyphae. Soluble dectin-1 (s-dectin-hFc) was used to visualize and (B) quantify $\beta$-glucan exposure above the signal threshold compared to the total area of the hyphae (C) mRNA abundance of $f k s A$, the 1,3-beta-glucan synthase catalytic subunit, and major subunit of glucan synthase using quantitative RT-PCR. All reactions were performed on BioRad MyIQ real-time PCR detection system with IQ SYBR green supermix. The $\Delta \Delta C_{t}$ method was used to combine 3 biological and 6 technical replicates for each transcript, using tefA as the housekeeping gene for normalization. *Indicates a $P$ value of $<0.05$ (unpaired two-tailed Students $t$ test) and fold change is relative to the normoxic control $=1.0$.

\subsection{Hypoxia leads to changes in overall cell wall composition and increased $\beta$-glucan levels}

To further define the changes in the cell wall induced by hypoxia, we next examined the alkali-insoluble (AI) and alkali-soluble (AS) fractions of the A. fumigatus cell wall in response to hypoxia. The AI-fraction largely consists of $\beta$ (1-3)-glucan, chitin, and galactomannan, while the ASfraction consists of $\alpha$-(1-3)-glucan and galactomannan [19]. Analysis of hypoxia cell wall composition supports the soluble dectin-1 staining and $f k s A$ mRNA transcription data and demonstrates that hypoxic growth is associated with increased levels of $\beta$-glucan in the AI-fraction and decreased levels of $\alpha$ glucan and galactosaminogalactan in the AS-fraction (Fig. 3A). The AS-fraction is generally present throughout the cell wall, but more in the outer face $[13,14]$. A decrease in the AS-fraction of the cell wall in hypoxia and an increase in the AI-fraction likely allows the components of the latter to be revealed, specifically, the $\beta$-glucan in that fraction (Fig. 3B). Thus, the higher amount of cell wall material found in the hypoxic grown mycelium correlates with increase in cell wall width observed in TEM images (Fig. 3 inset and 1D). Additionally there was an increase in $N$-acetylglucosamine (representative of the chitin content) in the AI-fraction in hypoxic-grown mycelia and a decrease in $N$-acetylgalactosamine in the AS-fraction of the hypoxic grown hyphae (Fig. 3A). Taken together, these results indicate that the stress of hypoxia causes alterations in the relative amounts and composition of A. fumigatus AS- and AI-fractions that are consistent with the observed thickening of the cell wall.

\subsection{Hypoxic-grown hyphae trigger an increased inflammatory response in bone marrow derived macrophages (BMDM) and neutrophils (BMDN)}

We next sought to determine if the hypoxia-induced changes in the fungal cell wall were immunologically relevant. Since macrophages act as initial responders to A. fumigatus in the lung and are important for controlling inflammatory responses, we co-cultured hypoxic- and normoxic-grown hyphae with BMDMs. After growth to defined morphological states, hyphae were inactivated by exposure to UV to eliminate the variable of pathogen growth during the fungal-host interaction and to avoid alterations in the fungal cell wall associated with heat-killing methods [40]. Hypoxic-grown hyphae were found to stimulate greater macrophage TNF and CXCL2 release compared to their normoxic counterparts (Fig. 4A and B). This response was not 
A

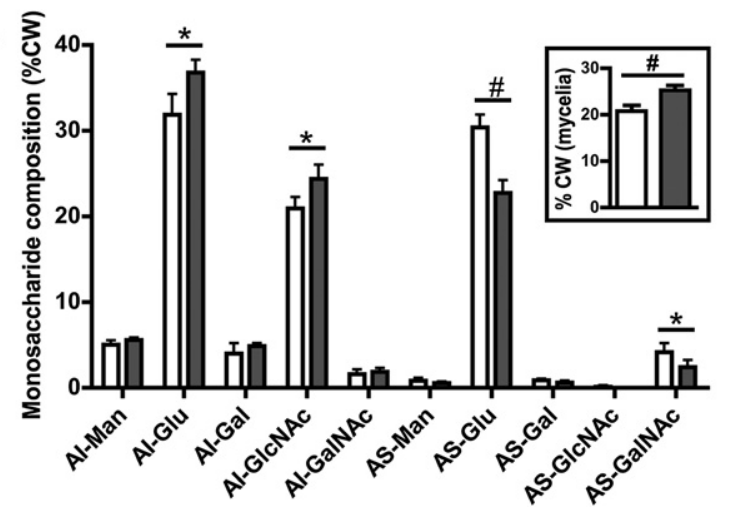

B

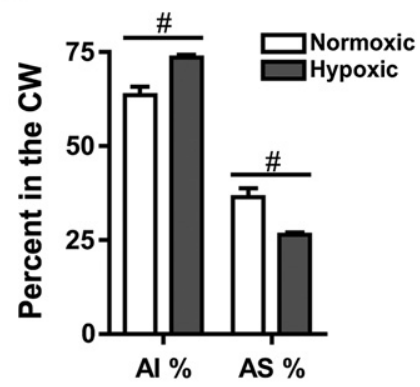

Fig. 3. Hypoxia causes changes in cell wall composition. (A) Monosaccharide composition of the alkali-insoluble (AI) and alkali-soluble (AS) fractions of CBS144.89 mycelial walls grown under normoxia and hypoxia for $24 \mathrm{~h}$ post germination. Results are expressed as percent of individual monosaccharides in the cell wall. Average percent of AI and AS in the cell wall (B) and the total amount of cell wall present (A inset) from the hypoxic and normoxic grown mycelial replicates. Values represent the average of five replicates plus SEM. *Indicates a $P$ value of $<0.05$ and \# a $P$ value of $<0.01$ (unpaired two-tailed Students $t$ test).

fungal strain specific as both A. fumigatus strains AF293 and CBS144.89 showed similar responses. Indeed, we have observed a similar response with hypoxia grown $C$. albicans (data not shown). In addition, a similar response was observed using murine macrophage cell lines J774.1 and RAW264.7 (data not shown). Importantly, the increased release of inflammatory mediators was also observed in murine BMDNs incubated with hypoxic hyphae (Fig. 4C). We chose to examine the response(s) to normoxic and hypoxic grown hyphae in multiple cell types to determine whether the response was cell-type specific. The conservation of response

A
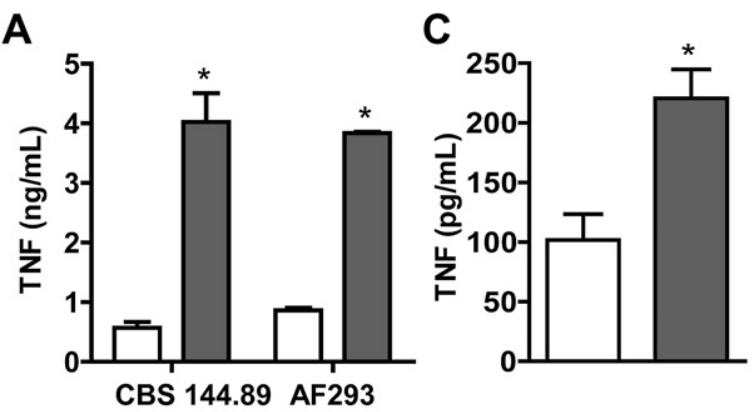

B
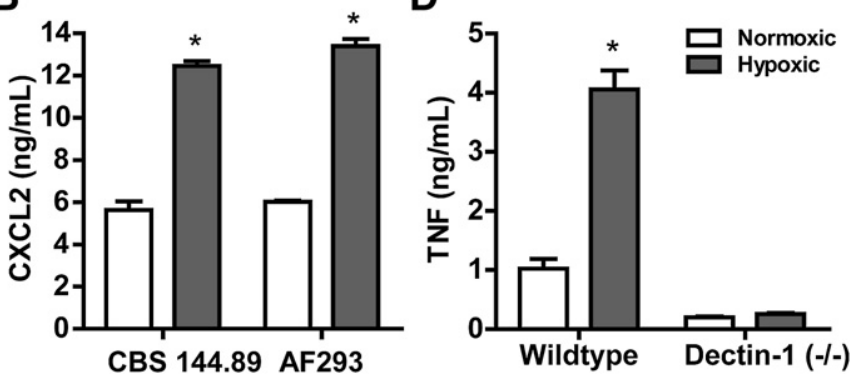

Fig. 4. Hypoxia-grown hyphae induce greater macrophage and neutrophil responsiveness than normoxia-grown hyphae. The bar graphs show the average $( \pm$ SEM) TNF (A, C, D) and CXCL2 (B) secretion by C57BL/6 (WT) (A, B, D) and dectin- $1^{(-l-)}$ (D) BMDMs and C57BL/6 (WT) BMDNs (C) in response to normoxia-grown (white bars) and hypoxia-grown (gray bars) hyphae as determined by ELISA. Fungal strains CBS144.89 (A, B, C, D) and AF293 (A, B) were examined. One of three representative experiments shown containing three biological replicates. ${ }^{*}$ Indicates a $P$ value of $<0.01$ (unpaired two-tailed Students $t$ test). in macrophage cell lines, BMDMs, and BMDNs, strongly suggests that the observed response is a general phenomenon in myeloid cells to hypoxic grown hyphae.

Previous studies have shown that macrophage TNF responses to A. fumigatus are largely mediated by dectin-1 engagement $[40,41]$. To determine if the increased macrophage response to hypoxic-grown hyphae was due to increased $\beta$-glucan exposure or to other changes in fungal cell wall components we compared TNF release by dectin-1-deficient and wild-type (C57BL/6) BMDMs stimulated with hypoxicgrown hyphae. TNF release was abrogated in the dectin-1deficient BMDMs, whether stimulated with hypoxia- or normoxia-grown hyphae (Fig. 4D). Thus, hypoxia-driven changes in the fungal cell wall content lead to greater macrophage and neutrophil inflammatory responses that are largely dependent on dectin-1 signaling.

\subsection{Increased macrophage antifungal activity against A. fumigatus with partial dectin-1 dependence}

Since hypoxic-grown hyphae induce greater macrophage and neutrophil cytokine and chemokine release, we next sought to quantitate phagocyte antifungal activity under normoxic and hypoxic conditions with live hyphae. Hyphal damage was assessed using the XTT hyphal damage assay, which measures fungal cell metabolic activity as an indirect measure of viability [35,36]. J774A.1 and RAW264.7 macrophage cell lines were incubated with normoxic grown $A$. fumigatus hyphae and co-cultured in hypoxia or normoxia for $2 \mathrm{~h}$. Macrophages induced greater hyphal damage when cocultured with A. fumigatus under hypoxic rather than normoxic conditions (Fig. 5A). We observed a similar response with primary human neutrophils (Fig. 5B). Additionally, we sought to determine the contribution of dectin- 1 in the antifungal response to hypoxic and normoxic grown hyphae using peritoneal macrophages isolated from C57BL/6 (WT) and dectin- $1^{(-/-)}$mice. Dectin-1 peritoneal macrophages induced less damage when co-cultured with A. fumigatus under hypoxic and normoxic conditions compared to WT peritoneal macrophages (Fig. 5C). Collectively these data demonstrate 
A

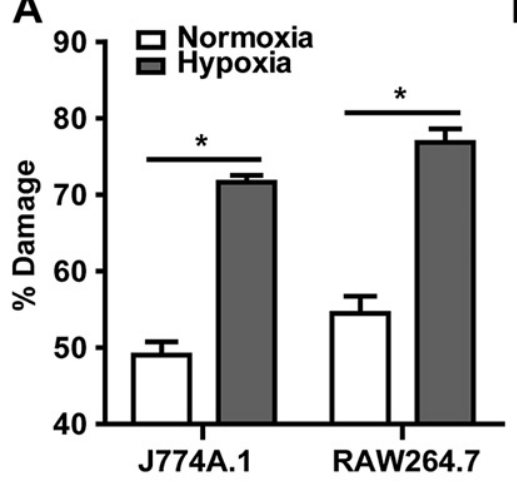

B

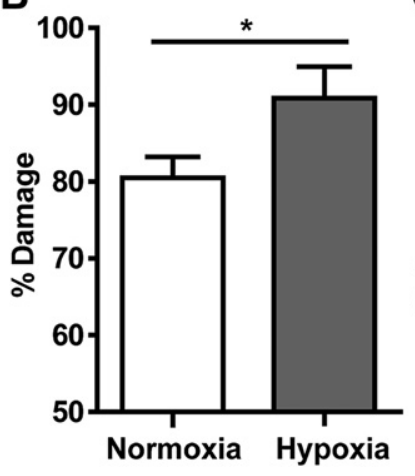

C

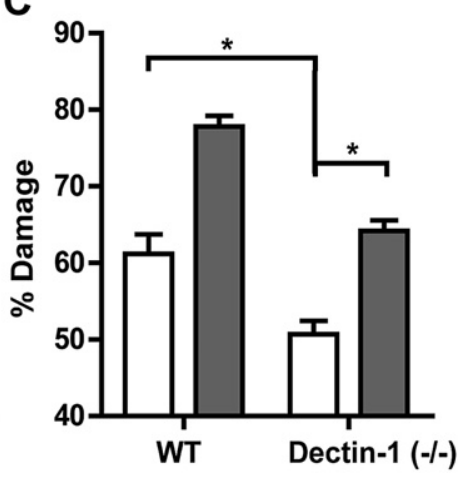

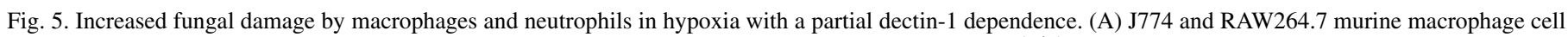

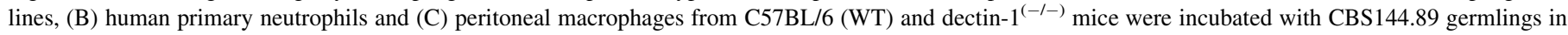

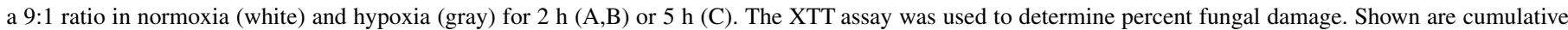
data from three independent studies. The data are expressed as plus SEM. *Indicates $P$ value of $<0.02$ (unpaired two-tailed Students $t$ test).
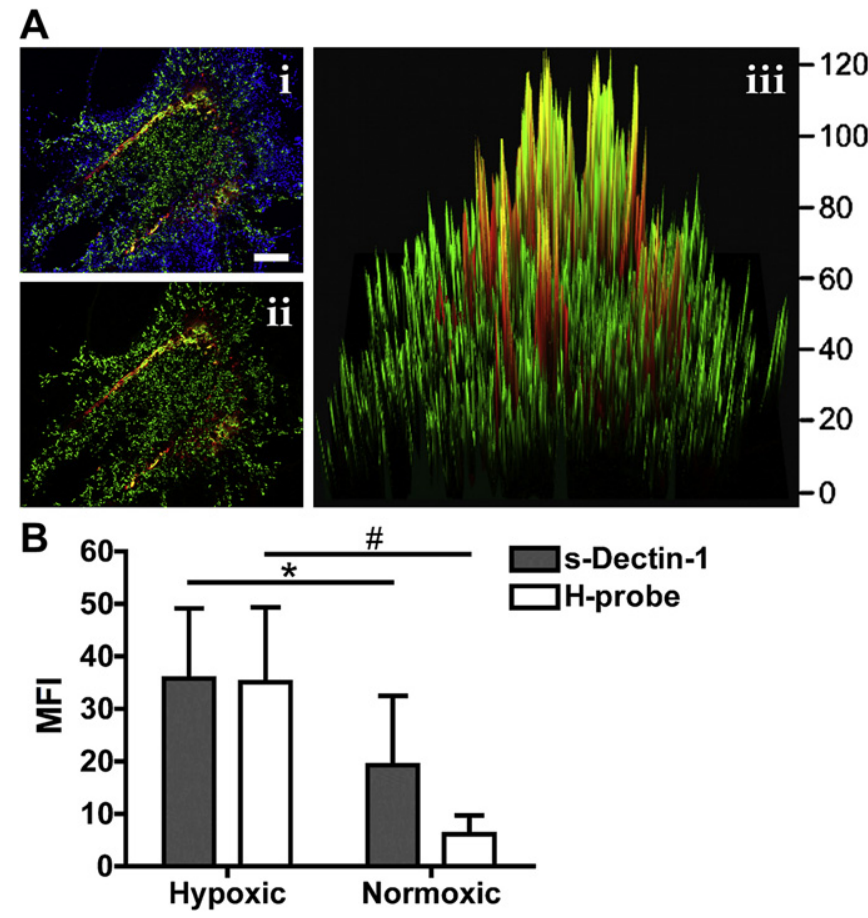

Fig. 6. In vivo hypoxia corresponds to increased $\beta$-glucan exposure in a corticosteroid model of IPA. Prior to sacrifice at the specified time point (day $3.5)$, hypoxyprobe-1 $(60 \mathrm{mg} / \mathrm{kg})$ was intravenously injected into the experimental mice shown and allowed to circulate for $60-90 \mathrm{~min}$. After the tissue was prepped, specific antibodies and proteins were used to detect hypoxyprobe- 1 bound proteins (red) and $\beta$-glucan (s-dectin-hFc, green). Host nuclei were counterstained with DAPI (blue). (A) Shown are representative pictures. Merged pictures consisted of all three colors (i) and only red and green (ii), that was used to generate the intensity plot (iii). Scale reflects the level of intensity. Scale bar $=100 \mu \mathrm{m}$. (B) Quantification of the level of the mean fluorescent intensity of the staining at sites of hypoxia. Two images were analyzed; (A) and data not shown. 5 Locations corresponding to hypoxic sites (h-probe staining) and 5 locations to non-hypoxic (no h-probe staining) were analyzed for the two figures. *Indicates $P$ value of $<0.03$ and \# indicates $P$ value of $<0.01$ (unpaired two-tailed Students $t$ test). (For interpretation of the references to colour in this figure legend, the reader is referred to the web version of this article.) that, under hypoxic conditions, immunocompetent phagocytes display increased cytokine and chemokine responses and induce greater hyphal damage that has partial dectin-1 dependence. Whether the increased fungal damage represents increased susceptibility of the fungal cells to phagocyte killing mechanisms under hypoxia, or novel phagocyte fungal killing mechanisms induced in hypoxia is currently unclear but under further investigation.

\section{6. $\beta$-Glucan exposure corresponds to regions of hypoxia in vivo in a corticosteroid model of IPA}

We next sought to determine the in vivo relevance of our in vitro findings related to fungal cell wall composition under hypoxia. To test this, we employed the use of the hypoxia marker, pimonidazole hydrochloride, which is activated by cellular mammalian nitroreductases and results in the formation of covalent adducts with thiol groups when oxygen levels are at $1.5 \%$ or below [42-45]. Results presented in Fig. 6 demonstrate that the level of soluble dectin-1 staining, measuring the amount of fungal $\beta$-glucan exposure, was greater in regions that co-stained with hypoxyprobe in our IPA murine model characterized by immunosuppression via use of the corticosteroid Triamcinolone (Aiii). $\beta$-Glucan staining was highest in regions with hypoxyprobe co-staining and decreased progressively when co-staining was not observed quantified through measurement of MFIs of hypoxic and nonhypoxic regions (Fig. 6B). Taken together, these results indicate that $A$. fumigatus undergoes cell wall remodeling in hypoxic microenvironments in vivo.

\section{Discussion}

Despite advances in the diagnosis and treatment of IFIs, mortality rates remain high and therapeutic options remain limited. One approach to improving patient outcomes associated with these devastating infections is to better understand the physiology of tissue microenvironments that develop during the fungal-host interaction. In this study we continue 
our exploration of the impact of hypoxia on A. fumigatus-mammalian interactions in the lung. We present findings that suggest hypoxia induces significant modifications in the fungal cell wall of $A$. fumigatus hyphae through unknown mechanisms and that these changes can subsequently affect innate phagocytic cell responses. Thus, we believe our data has potential clinical relevance and could potentially be utilized in the future to improve therapeutic options for IPA as we discuss below.

With regard to affecting the outcome of IPA, our data suggest that hypoxia-mediated modifications of the cell wall impact both the physiology of the fungus and host. First, our data correlates with previous results that demonstrate that stress, whether drug [25,46] or environmental induced [22], leads to increased exposure of $\beta$-glucan, a major fungal PAMP, on the hyphal surface of A. fumigatus, C. albicans, and other pathogenic fungi. The mechanism behind the increase in $\beta$ glucan exposure is not understood, but may reflect a general stress response, as it has now been observed in different fungal species and with multiple stressors (7, 18-20, 23, 28, 29, 41, 48-50). We did observe a significant increase in fksA mRNA abundance in response to hypoxia, but how this translates to FksA enzyme activity and thus $\beta$-glucan production is unknown. While hypoxia appears to reduce overall fungal metabolism, cell wall biosynthesis is an energy intensive biosynthetic process that involves several metabolic pathways such as glycolysis that are affected by hypoxia [18]. Future biochemical studies are needed to correlate changes in mRNA abundance in these pathways and metabolites needed for cell wall biosynthesis.

Importantly, our in vitro observations related to increased $\beta$-glucan exposure on hypoxia-exposed hyphae were also observed in vivo in our corticosteroid model of IPA. Autopsyand animal model-based studies indicate that the pathogenesis of IA differs markedly among neutropenic and glucocorticoidtreated, non-neutropenic hosts (reviewed in [47]). For example, it has been proposed that mortality in corticosteroid models of IPA stems largely from a hyperinflammatory response to the mold rather than direct fungal growth per se [48]. Our ex vivo data provides indirect support for this observation and potentially correlates this finding with hypoxia driven changes to the fungal cell wall as we observed significant increase in pro-inflammatory cytokine production from murine cell line and BM-derived macrophages and neutrophils exposed to hypoxia grown hyphae. This increased responsiveness is at least partially due to the increased exposure of $\beta$-glucan as previous studies have shown the correlation between $\beta$-glucan exposure and magnitude of cytokine responses [25]. While this result appears largely dectin-1dependent, and thus correlated with increased $\beta$-glucan exposure, we cannot rule out that other changes in the cell wall induced by hypoxia affect phagocytic cell responses. Indeed, growing evidence suggests that multiple components of the $A$. fumigatus and other fungi cell walls have immunomodulatory activity $[14,16,49,50]$.

Along these lines of thinking, our in vitro and in vivo findings that related in vivo hypoxia to enhanced phagocyte responses may also relate to the clinical and radiographic observation that fungal lesions increase in size and can lead to symptomatic deterioration at the time of neutrophil recovery. IRIS is one of the factors associated with deaths caused by fungal infections [31] and is thought to occur due to an uncontrolled, pathogen-driven immune response and a failure of regulatory responses to control inflammation [31]. Our work predicts that under these situations where hypoxic microenvironments are established in lung lesions, enhanced $\beta$-(1,3)-glucan exposure and other cell wall changes driven by hypoxia may augment phagocyte responsiveness beyond optimal levels required for fungal eradication. This scenario would contribute to the development of immunopathology and subsequent poor treatment outcomes. Conversely, $\beta$-glucan exposure could possibly be beneficial to the host, permitting a more directed immune response in hypoxic areas of the lung and perhaps activating more effective mechanisms of fungal killing by phagocytes. The balance between beneficial and detrimental immune stimulation in different host environments found during IPA remains an important area of investigation. Along these lines, it will be important to determine the mechanisms in play behind the observed increased fungal damage caused by phagocytes under hypoxia.

In conclusion, we present data suggesting that in vivo hypoxia alters A. fumigatus cell wall dynamics that in turn affect the pathogenesis of IPA. These findings naturally lead to translational research questions that we will pursue in future studies. For example, if hypoxia helps drive immunopathology during neutrophil recovery and/or in non-neutropenic patients with an A. fumigatus or other fungal infections (such as chronic granulomatous disease patients or those on corticosteroid therapies), can modulation of oxygen levels at the site of infection reverse this host mediated damage? In addition, can regulators of the host hypoxia response be targeted via existing drugs to reduce adverse inflammatory responses? And finally, we observed that hypoxia actually increases the ability of immunocompetent phagocytes to damage A. fumigatus hyphae, and thus in certain patients, induction of the hypoxia response may actually be beneficial. Thus, we believe that further study of fungal and host mediated hypoxia responses in clinically relevant models of IPA have great potential to lead to new therapeutic strategies for IPA and further our understanding of this important infectious disease.

\section{Acknowledgments}

This work was supported by a grant from the National Center for Research Resources (RR020185, M. Quinn PI, RAC project 2 leader) and National Institute of General Medicine (P20GM103500) from the National Institutes of Health (NIH), NIH/NIAID R01AI81838 to RAC, and the Montana State University Agricultural Experiment Station (RAC). This work was also partially supported by a HHMI/ UW Molecular Medicine Program clerkship, the Robert Sinskey Foundation, and NIH R01AI093808 (TH). This research was supported by grants from MEXT, CREST and the Promotion of Basic Research Activities for Innovative 
Biosciences Program (YI). KSM was partially supported by a Molecular Biosciences Fellowship at Montana State University-Bozeman.

The authors would like to thank Kiyoshi Ariizumi (University of Texas Southwestern Medical Center) for the soluble dectin-1-Fc construct. Members of the Cramer laboratory, especially Dr. Nora Grahl, the Hohl Laboratory, especially Debra Kumasaka, for reagents and useful comments on the manuscript. We would also like to thank the Harmsen laboratory, and the Jovanka Voyich (NIH/NIAID R01AI090046) laboratory, especially Mark Dewald, in the department of Immunology and Infectious Diseases at Montana State University for use of resources related to the hypoxyprobe, immunology studies, and isolation of human neutrophils (JV/MD).

\section{References}

[1] B.H. Segal, Aspergillosis, N. Engl. J. Med. 360 (2009) 1870-1884.

[2] A. Upton, K.A. Kirby, P. Carpenter, M. Boeckh, K.A. Marr, Invasive aspergillosis following hematopoietic cell transplantation: outcomes and prognostic factors associated with mortality, Clin. Infect. Dis. 44 (2007) 531-540.

[3] S.J. Howard, M.C. Arendrup, Acquired antifungal drug resistance in Aspergillus fumigatus: epidemiology and detection, Med. Mycol. 49 (Suppl. 1) (2011) S90-S95.

[4] Z. Erjavec, H. Kluin-Nelemans, P.E. Verweij, Trends in invasive fungal infections, with emphasis on invasive aspergillosis, Clin. Microbiol. Infect. 15 (2009) 625-633.

[5] J.W. Bok, N.P. Keller, D.I. Tsitsigiannis, Real-time and semiquantitative RT-PCR methods to analyze gene expression patterns during Aspergillushost interactions, Methods Mol. Biol. 470 (2009) 151-167.

[6] N.M. Cooney, B.S. Klein, Fungal adaptation to the mammalian host: it is a new world, after all, Curr. Opin. Microbiol. 11 (2008) 511-516.

[7] T. Hartmann, C. Sasse, A. Schedler, M. Hasenberg, M. Gunzer, S. Krappmann, Shaping the fungal adaptome - stress responses of Aspergillus fumigatus, Int. J. Med. Microbiol. 301 (2011) 408-416.

[8] R.S. Shapiro, N. Robbins, L.E. Cowen, Regulatory circuitry governing fungal development, drug resistance, and disease, Microbiol. Mol. Biol. Rev. 75 (2011) 213-267.

[9] R. Bhabhra, D.S. Askew, Thermotolerance and virulence of Aspergillus fumigatus: role of the fungal nucleolus, Med. Mycol. 43 (Suppl. 1) (2005) S87-S93.

[10] N. Grahl, S. Puttikamonkul, J.M. Macdonald, M.P. Gamcsik, L.Y. Ngo, T.M. Hohl, R.A. Cramer, In vivo hypoxia and a fungal alcohol dehydrogenase influence the pathogenesis of invasive pulmonary aspergillosis, PLoS Pathog. 7 (2011) e1002145.

[11] S.D. Willger, S. Puttikamonkul, K.H. Kim, J.B. Burritt, N. Grahl, L.J. Metzler, R. Barbuch, M. Bard, C.B. Lawrence, R.A. Cramer Jr., A sterol-regulatory element binding protein is required for cell polarity, hypoxia adaptation, azole drug resistance, and virulence in Aspergillus fumigatus, PLoS Pathog. 4 (2008) e1000200.

[12] M. Blatzer, B.M. Barker, S.D. Willger, N. Beckmann, S.J. Blosser, E.J. Cornish, A. Mazurie, N. Grahl, H. Haas, R.A. Cramer, SREBP coordinates iron and ergosterol homeostasis to mediate triazole drug and hypoxia responses in the human fungal pathogen Aspergillus fumigatus, PLoS Genet. 7 (2011) e1002374.

[13] J.P. Latge, The cell wall: a carbohydrate armour for the fungal cell, Mol. Microbiol. 66 (2007) 279-290.

[14] J.P. Latge, Tasting the fungal cell wall, Cell. Microbiol. 12 (2010) $863-872$.

[15] C.A. Janeway Jr., R. Medzhitov, Innate immune recognition, Annu. Rev. Immunol. 20 (2002) 197-216.

[16] T. Fontaine, A. Delangle, C. Simenel, B. Coddeville, S.J. van Vliet, Y. van Kooyk, S. Bozza, S. Moretti, F. Schwarz, C. Trichot, M. Aebi, M. Delepierre, C. Elbim, L. Romani, J.P. Latge, Galactosaminogalactan, a new immunosuppressive polysaccharide of Aspergillus fumigatus, PLoS Pathog. 7 (2011) e1002372.

[17] N. Abramova, O. Sertil, S. Mehta, C.V. Lowry, Reciprocal regulation of anaerobic and aerobic cell wall mannoprotein gene expression in Saccharomyces cerevisiae, J. Bacteriol. 183 (2001) 2881-2887.

[18] B.M. Barker, K. Kroll, M. Vodisch, A. Mazurie, O. Kniemeyer, R.A. Cramer, Transcriptomic and proteomic analyses of the Aspergillus fumigatus hypoxia response using an oxygen-controlled fermenter, BMC Genom. 13 (2012) 62.

[19] A. Gastebois, C. Clavaud, V. Aimanianda, J.P. Latge, Aspergillus fumigatus: cell wall polysaccharides, their biosynthesis and organization, Future Microbiol. 4 (2009) 583-595.

[20] J.G. Gibbons, A. Beauvais, R. Beau, K.L. McGary, J.P. Latge, A. Rokas, Global transcriptome changes underlying colony growth in the opportunistic human pathogen Aspergillus fumigatus, Eukaryot. Cell 11 (2012) $68-78$.

[21] E. Mellado, G. Dubreucq, P. Mol, J. Sarfati, S. Paris, M. Diaquin, D.W. Holden, J.L. Rodriguez-Tudela, J.P. Latge, Cell wall biogenesis in a double chitin synthase mutant (chsG-/chsE-) of Aspergillus fumigatus, Fungal Genet. Biol. 38 (2003) 98-109.

[22] G.J. Sosinska, P.W. de Groot, M.J. Teixeira de Mattos, H.L. Dekker, C.G. de Koster, K.J. Hellingwerf, F.M. Klis, Hypoxic conditions and iron restriction affect the cell-wall proteome of Candida albicans grown under vagina-simulative conditions, Microbiology 154 (2008) 510-520.

[23] R.S. Zitomer, P. Carrico, J. Deckert, Regulation of hypoxic gene expression in yeast, Kidney Int. 51 (1997) 507-513.

[24] L. Zhao, D. Schaefer, H. Xu, S.J. Modi, W.R. LaCourse, M.R. Marten, Elastic properties of the cell wall of Aspergillus nidulans studied with atomic force microscopy, Biotechnol. Prog. 21 (2005) 292-299.

[25] T.M. Hohl, M. Feldmesser, D.S. Perlin, E.G. Pamer, Caspofungin modulates inflammatory responses to Aspergillus fumigatus through stage-specific effects on fungal beta-glucan exposure, J. Infect. Dis. 198 (2008) 176-185.

[26] G.A. Lamaris, R.E. Lewis, G. Chamilos, G.S. May, A. Safdar, T.J. Walsh, I.I. Raad, D.P. Kontoyiannis, Caspofungin-mediated beta-glucan unmasking and enhancement of human polymorphonuclear neutrophil activity against Aspergillus and non-Aspergillus hyphae, J. Infect. Dis. 198 (2008) 186-192.

[27] G.D. Brown, P.R. Taylor, D.M. Reid, J.A. Willment, D.L. Williams, L. Martinez-Pomares, S.Y. Wong, S. Gordon, Dectin-1 is a major betaglucan receptor on macrophages, J. Exp. Med. 196 (2002) 407-412.

[28] K.M. Dennehy, G.D. Brown, The role of the beta-glucan receptor Dectin1 in control of fungal infection, J. Leukoc. Biol. 82 (2007) 253-258.

[29] D.L. Barber, K.D. Mayer-Barber, L.R. Antonelli, M.S. Wilson, S. White, P. Caspar, S. Hieny, I. Sereti, A. Sher, Th1-driven immune reconstitution disease in Mycobacterium avium-infected mice, Blood 116 (2010) 3485-3493.

[30] A.O. Gupta, N. Singh, Immune reconstitution syndrome and fungal infections, Curr. Opin. Infect. Dis. 24 (2011) 527-533.

[31] M.H. Miceli, J. Maertens, K. Buve, M. Grazziutti, G. Woods, M. Rahman, B. Barlogie, E.J. Anaissie, Immune reconstitution inflammatory syndrome in cancer patients with pulmonary aspergillosis recovering from neutropenia: proof of principle, description, and clinical and research implications, Cancer 110 (2007) 112-120.

[32] L.M. Graham, S.V. Tsoni, J.A. Willment, D.L. Williams, P.R. Taylor, S. Gordon, K. Dennehy, G.D. Brown, Soluble Dectin-1 as a tool to detect beta-glucans, J. Immunol. Methods 314 (2006) 164-169.

[33] K.J. Livak, T.D. Schmittgen, Analysis of relative gene expression data using real-time quantitative PCR and the 2(-delta delta $\mathrm{C}(\mathrm{T})$ ) method, Methods 25 (2001) 402-408.

[34] D.L. Richie, L. Hartl, V. Aimanianda, M.S. Winters, K.K. Fuller, M.D. Miley, S. White, J.W. McCarthy, J.P. Latge, M. Feldmesser, J.C. Rhodes, D.S. Askew, A role for the unfolded protein response (UPR) in virulence and antifungal susceptibility in Aspergillus fumigatus, PLoS Pathog. 5 (2009) e1000258.

[35] J. Meletiadis, J.W. Mouton, J.F. Meis, B.A. Bouman, J.P. Donnelly, P.E. Verweij, Colorimetric assay for antifungal susceptibility testing of Aspergillus species, J. Clin. Microbiol. 39 (2001) 3402-3408. 
[36] T. Meshulam, S.M. Levitz, L. Christin, R.D. Diamond, A simplified new assay for assessment of fungal cell damage with the tetrazolium dye, (2,3)-bis-(2-methoxy-4-nitro-5-sulphenyl)-(2H)-tetrazolium-5-carboxanilide (XTT), J. Infect. Dis. 172 (1995) 1153-1156.

[37] J.M. Voyich, K.R. Braughton, D.E. Sturdevant, A.R. Whitney, B. SaidSalim, S.F. Porcella, R.D. Long, D.W. Dorward, D.J. Gardner, B.N. Kreiswirth, J.M. Musser, F.R. DeLeo, Insights into mechanisms used by Staphylococcus aureus to avoid destruction by human neutrophils, J. Immunol. 175 (2005) 3907-3919.

[38] W.J. Steinbach, D.K. Benjamin Jr., S.A. Trasi, J.L. Miller, W.A. Schell, A.K. Zaas, W.M. Foster, J.R. Perfect, Value of an inhalational model of invasive aspergillosis, Med. Mycol. 42 (2004) 417-425.

[39] K. Ariizumi, G.L. Shen, S. Shikano, S. Xu, R. Ritter 3rd, T. Kumamoto, D. Edelbaum, A. Morita, P.R. Bergstresser, A. Takashima, Identification of a novel, dendritic cell-associated molecule, dectin-1, by subtractive cDNA cloning, J. Biol. Chem. 275 (2000) 20157-20167.

[40] T.M. Hohl, H.L. Van Epps, A. Rivera, L.A. Morgan, P.L. Chen, M. Feldmesser, E.G. Pamer, Aspergillus fumigatus triggers inflammatory responses by stage-specific beta-glucan display, PLoS Pathog. 1 (2005) e30.

[41] C. Steele, R.R. Rapaka, A. Metz, S.M. Pop, D.L. Williams, S. Gordon, J.K. Kolls, G.D. Brown, The beta-glucan receptor dectin-1 recognizes specific morphologies of Aspergillus fumigatus, PLoS Pathog. 1 (2005) e42.

[42] J.A. Raleigh, A.J. Franko, C.J. Koch, J.L. Born, Binding of misonidazole to hypoxic cells in monolayer and spheroid culture: evidence that a sidechain label is bound as efficiently as a ring label, Br. J. Cancer 51 (1985) 229-235.

[43] J.A. Raleigh, D.P. Calkins-Adams, L.H. Rinker, C.A. Ballenger, M.C. Weissler, W.C. Fowler Jr., D.B. Novotny, M.A. Varia, Hypoxia and vascular endothelial growth factor expression in human squamous cell carcinomas using pimonidazole as a hypoxia marker, Cancer Res. 58 (1998) 3765-3768.

[44] A.S. Ljungkvist, J. Bussink, P.F. Rijken, J.A. Raleigh, J. Denekamp, A.J. Van Der Kogel, Changes in tumor hypoxia measured with a double hypoxic marker technique, Int. J. Radiat. Oncol. Biol. Phys. 48 (2000) $1529-1538$.

[45] G.E. Arteel, R.G. Thurman, J.A. Raleigh, Reductive metabolism of the hypoxia marker pimonidazole is regulated by oxygen tension independent of the pyridine nucleotide redox state, Eur. J. Biochem. 253 (1998) 743-750.

[46] R.T. Wheeler, D. Kombe, S.D. Agarwala, G.R. Fink, Dynamic, morphotype-specific Candida albicans beta-glucan exposure during infection and drug treatment, PLoS Pathog. 4 (2008) e1000227.

[47] R.E. Lewis, D.P. Kontoyiannis, Invasive aspergillosis in glucocorticoidtreated patients, Med. Mycol. 47 (Suppl. 1) (2009) S271-S281.

[48] V. Balloy, M. Huerre, J.P. Latge, M. Chignard, Differences in patterns of infection and inflammation for corticosteroid treatment and chemotherapy in experimental invasive pulmonary aspergillosis, Infect. Immun. 73 (2005) 494-503.

[49] L.Y. Chai, A.G. Vonk, B.J. Kullberg, P.E. Verweij, I. Verschueren, J.W. van der Meer, L.A. Joosten, J.P. Latge, M.G. Netea, Aspergillus fumigatus cell wall components differentially modulate host TLR2 and TLR4 responses, Microbe. Infect. 13 (2011) 151-159.

[50] H.M. Mora-Montes, M.G. Netea, G. Ferwerda, M.D. Lenardon, G.D. Brown, A.R. Mistry, B.J. Kullberg, C.A. O'Callaghan, C.C. Sheth, F.C. Odds, A.J. Brown, C.A. Munro, N.A. Gow, Recognition and blocking of innate immunity cells by Candida albicans chitin, Infect. Immun. 79 (2011) 1961-1970. 